\title{
FRACTAL STRICHARTZ ESTIMATE FOR THE WAVE EQUATION
}

\author{
CHU-HEE CHO, SEHEON HAM, AND SANGHYUK LEE
}

\begin{abstract}
We consider Strichartz estimates for the wave equation with respect to general measures which satisfy certain growth conditions. In $\mathbb{R}^{3+1}$ we obtain the sharp estimate and in higher dimensions improve the previous results.
\end{abstract}

\section{INTRODUCTION}

Let us consider the wave equation in $\mathbb{R}^{n} \times \mathbb{R}$ :

$$
\left\{\begin{array}{l}
\partial_{t}^{2} u-\Delta u=0, \\
u(x, 0)=f, \quad \partial_{t} u(x, 0)=g .
\end{array}\right.
$$

The space-time estimate for the solution of (1) which is called Strichartz estimate has been proven to be an important tool in studies of various problems. (See [11,12, 14, 18, 21,22.) It is well-known that the estimate

$$
\|u\|_{L_{t}^{q}\left(\mathbb{R}, L_{x}^{r}\left(\mathbb{R}^{n}\right)\right)} \lesssim\|f\|_{\dot{H}^{s}}+\|g\|_{\dot{H}^{s-1}}
$$

holds for $s \geq 0,2 \leq q, r<\infty$ which satisfy

$$
\frac{1}{q}+\frac{n}{r}=\frac{n}{2}-s, \frac{1}{q}+\frac{n-1}{2 r} \leq \frac{n-1}{4} .
$$

Here $\dot{H}^{s}$ is the homogeneous $L^{2}$ Sobolev space of order $s$. See 7 for the estimates with $r=\infty$. It was Strichartz [22] who first proved the estimate (2) when $q=r$. This was later extended to mixed norm estimates by Pecher [21]. (Also see [8].) The endpoint cases $(r, q)=(2(n-1) /(n-3), 2)$ except $n=3$ were obtained by Keel-Tao [11]. Klainerman and Machedon [12] showed the failure of (2) when $(n, r, q)=(3, \infty, 2)$.

In this note we consider a generalization of (2) by replacing the Lebesgue measure with general measure $\mu$. More precisely, we study the estimate

$$
\|u\|_{L^{q}(d \mu)} \lesssim\|f\|_{H^{s}}+\|g\|_{H^{s-1}} .
$$

Here we denote by $H^{s}\left(\mathbb{R}^{n}\right)$ the inhomogeneous $L^{2}$ Sobolev space of order $s$, which is the space of all tempered distributions $f$ such that $\left(1+|\cdot|^{2}\right)^{\frac{s}{2}} \widehat{f} \in L^{2}\left(\mathbb{R}^{n}\right)$, equipped

\footnotetext{
Key words and phrases. wave equation, Strichartz estimate, general measure.

This work is supported by NRF-2015R1A4A1041675 and NRF- 2015R1A2A2A05000956 (South Korea).
} 
with the norm

$$
\|f\|_{H^{s}\left(\mathbb{R}^{n}\right)}=\left\|\left(1+|\cdot|^{2}\right)^{\frac{s}{2}} \widehat{f}\right\|_{L^{2}\left(\mathbb{R}^{n}\right)} .
$$

This kind of estimates was studied in connection with problems in geometric measure theory, precisely, the sphere packing problem (see [17, 19, 20, 27]).

Throughout this paper, the measure $\mu$ is assumed to be a nonnegative Borel regular measure with compact support in $\mathbb{R}^{n+1}$. Let us denote by $\mathfrak{M}\left(\mathbb{R}^{n+1}\right)$ the space of such measures. In addition we impose uniform growth condition on $\mu$ as follows.

Definition 1.1. Let $\alpha \in(0, n+1]$. For $\mu \in \mathfrak{M}\left(\mathbb{R}^{n+1}\right)$, we say that $\mu$ is $\alpha$-dimensional if there exists a constant $C_{\mu}$, independent of $x$ and $\rho$, such that

$$
\mu(B(x, \rho)) \leq C_{\mu} \rho^{\alpha} \quad \text { for all } x \in \mathbb{R}^{n+1}, \rho>0 .
$$

Here $B(x, \rho)$ denotes the open ball of radius $\rho$ centered at $x$. Also we define

$$
\langle\mu\rangle_{\alpha}=\sup _{x \in \mathbb{R}^{n+1}, \rho>0} \rho^{-\alpha} \mu(B(x, \rho)) .
$$

For $1 \leq q \leq \infty$ let us set

$$
s(\alpha, q, n)= \begin{cases}\max \left\{\frac{n}{2}-\frac{\alpha}{q}, \frac{n+1}{4}\right\}, & \text { if } 0<\alpha \leq 1, \\ \max \left\{\frac{n}{2}-\frac{\alpha}{q}, \frac{n+1}{4}+\frac{1-\alpha}{2 q}, \frac{n+2}{4}-\frac{\alpha}{4}\right\}, & \text { if } 1<\alpha \leq n, \\ \max \left\{\frac{n}{2}-\frac{\alpha}{q}, \frac{n+1}{4}+\frac{n+1-2 \alpha}{2 q}, \frac{n+1}{2}-\frac{\alpha}{2}\right\}, & \text { if } n<\alpha \leq n+1 .\end{cases}
$$

When $n=2$ Wolff [27] showed that (3) holds for $\alpha$-dimensional measure $\mu$ if $s>$ $\max \left(\frac{3}{4}, 1-\frac{\alpha}{4}, 1-\frac{\alpha}{q}\right), \alpha \in(1,3)$. Erdoğan [4] improved Wolff's result so that (3) holds for $s>s(\alpha, q, 2), \alpha \in(1,3)$ and also showed that (3) generally fails if $s<s(\alpha, q, 2)$. When $n \geq 3$, Oberlin [19] obtained (3) for $\alpha \in(1, n+1)$ provided that $q<\alpha$ and $s>\frac{n-1}{2}$.

It is plausible to conjecture that (3) holds if $s>s(\alpha, q, n)$ (see Proposition 1.5) but like other open problems of similar nature complete resolution seems out of reach at this moment. However, for $n=3$ and $\alpha \in[1,4]$, we obtain the sharp estimate by the following theorem and Proposition 1.5,

Theorem 1.2. Let $n=3$. Also let $\mu$ be an $\alpha$-dimensional measure. Suppose that $u$ is a solution to the equation (11). Then (3) holds with

$$
s>\left\{\begin{array}{l}
s(\alpha, q, 3), \quad \text { if } 2 \leq q \leq \infty, \\
s(\alpha, 2,3), \quad \text { if } 1 \leq q \leq 2
\end{array}\right.
$$

Furthermore, the implicit constant in (3) does not depend on particular choice of $\mu$ as long as $\langle\mu\rangle_{\alpha}$ is uniformly bounded.

For $\delta>0$ and $R \gg 1$, let us define the truncated cone $\Gamma_{R}$ by

$$
\Gamma_{R}=\left\{\xi=\left(\xi^{\prime}, \xi_{n+1}\right) \in \mathbb{R}^{n+1}:\left|\xi^{\prime}\right|=\xi_{n+1}, R \leq \xi_{n+1} \leq 2 R\right\}
$$


and its $\delta$-neighborhood by

$$
\Gamma_{R}(\delta)=\left\{\xi \in \mathbb{R}^{n+1}: \operatorname{dist}\left(\xi, \Gamma_{R}\right)<\delta\right\} .
$$

Note that the space-time Fourier transform of $u$ is supported in the forward and backward light cones. By reflection in frequency spaces, Littlewood-Paley decomposition, and Plancherel theorem, Theorem 1.2 is a consequence of the following. (For details, see the end of Section 3.)

Theorem 1.3. Let $n=3$ and $\mu$ be an $\alpha$-dimensional measure supported in $\overline{B(0,1)}$. Then, for $f$ supported in $\Gamma_{R}(1)$ there exists a constant $C>0$ such that

$$
\|\widehat{f}\|_{L^{q}(d \mu)} \leq C\langle\mu\rangle_{\alpha}^{\frac{1}{q}} R^{s}\|f\|_{2}
$$

holds if $s$ satisfies (6).

Remark 1.4. If $n=3$ and $\alpha \in[1,4]$, (17) is sharp because $s(\alpha, q, 3)=s(\alpha, 2,3)$ when $1 \leq q \leq 2$. If $0<\alpha<1$, we have $s(\alpha, 2,3)>s(\alpha, q, 3)$ for $1 \leq q \leq 2$.

In general, $s(\alpha, q, n), n \geq 3$ provides a lower bound on $s$ for (7) to hold.

Proposition 1.5. Suppose that for any $\alpha$-dimensional measure $\mu$, there is a constant $C>0$ such that (7) holds whenever $f$ is supported in $\Gamma_{R}(1) \subset \mathbb{R}^{n+1}$. Then $s \geq$ $s(\alpha, q, n)$ for $1 \leq q \leq \infty$.

In order to show this, we construct $\alpha$-dimensional measures and functions for which (17) fails if $s<s(\alpha, q, n)$. For $\alpha \in(1, n+1]$, we modify the examples in [4 and for $\alpha \in(0,1]$ we use the construction in [10]. (See Section 4 for details.) For each $\alpha$ some of the conditions appearing in (5) are natural in view of dilation and rescaling structure of the estimate (7). To be precise, for the case $n<\alpha \leq n+1$ in (5) the condition $s(\alpha, q, n)=\frac{n+1}{4}+\frac{n+1-2 \alpha}{2 q}$ may be understood as a homogeneity condition and $s(\alpha, q, n)=\frac{n}{2}-\frac{\alpha}{q}$ is related to the Knapp type example. A similar statement also applies to the case $1<\alpha \leq n$.

In contrast with the Lebesgue measure, there is no obvious scaling structure for a general $\alpha$-dimensional measure. But, as is to be seen in what follows, if we assume uniform bound which only depends on $\langle\mu\rangle_{\alpha}$, the measure $\mu$ can be handled as if it is homogeneous of degree $\alpha$ with respect to isotropic dilation. This observation plays an important role throughout the paper. Similar idea was used in [10] to obtain restriction estimate for the curve with respect to general measures other than the Lebesgue measure.

The estimate (7) is closely related to Fourier restriction problem. We make use of the bilinear approach which was extensively utilized to tackle the restriction problem. Some aspects of our paper are similar to those of [4]. However, unlike [4] we use the induction on scale argument in more direct way without relying on weighted inequality and this enables us to expose underlying structure more clearly. Also our argument can be used to recover the results in [4]. It is natural to expect that multilinear restriction estimates [1] and its recent development (see [3, 9]) can be used 
for further improvement of the estimate (3). However, it does not seem likely that these estimates would give the sharp estimate such as our result in Theorem 1.2 .

In this paper we will prove (7) for $n \geq 3$ (see Theorem 3.1) while our results are sharp only for $n=3$. (The sharp estimates for $n=2$ and $1 \leq \alpha \leq 3$ was previously obtained by Erdoğan [4].) When $n \geq 4$, the necessary condition $s \geq s(\alpha, q, n)$ (except the equality case) is sufficient only for $n<\alpha \leq n+1$ or for large $q$ if $\alpha \leq n$.

Average decay estimate. It is well-known that (7) implies the associated average decay estimate. In fact the decay rate is determined by $s(=s(\alpha, 2, n))$ of the estimate (17). Let $I_{\alpha}(\mu)$ be an $\alpha$-dimensional energy of $\mu$ which is given by $I_{\alpha}(\mu)=\iint \mid x-$ $\left.y\right|^{-\alpha} d \mu(x) d \mu(y)$. If $\mu$ is a positive Borel measure supported in the unit ball and satisfies $I_{\alpha}(\mu)=1$, there exists a constant $C_{\alpha}>0$ such that

$$
\int_{\Gamma_{1}}|\widehat{\mu}(R \xi)|^{2} d \sigma(\xi) \leq C_{\alpha} R^{-n+2 s(\alpha, 2,3)+\varepsilon}
$$

for $R>1$ and any $\varepsilon>0$. This can be shown by the argument in [26] which makes use of Lemma 1.5 and duality. (See Section 2 in [26] for details.)

The average decay estimate over the sphere has been studied in connection with Falconer's distance set conjecture. (See [16], [2], [26], [5], 6], [15] and references therein.)

The sphere packing problem. Let $S(x, r)$ be a sphere in $\mathbb{R}^{n}$ with center $x$ and radius $r>0$. We denote the Hausdorff dimension by $\operatorname{dim}_{H}$ and the $d$-dimensional Lebesgue measure by $|\cdot|_{d}$. Theorem 1.3 immediately implies the following.

Corollary 1.6. Let $E \subset \mathbb{R}^{n}$ and $P$ be a Borel set in $\mathbb{R}^{n+1}$ with $\operatorname{dim}_{H}(P)>1$. Assume that $|S(x, r) \cap E|_{n-1}>0$ for any $(x, r) \in P$. Then $|E|_{n}>0$.

Wolff [27] proved that Corollary 1.6 is valid when $n=2$. When $n \geq 3$, Oberlin [19] showed that the statement holds by obtaining estimate for the spherical average.

Organization of the paper. In Section 2 we prove a bilinear version of (7), which is obtained by an adaptation of the induction on scale argument. In Section 3 proofs of Theorem 1.3 and Theorem 1.2 are given. In Section 4 , we discuss the necessary conditions in Proposition 1.5.

\section{Bilinear estimates}

In this section we prove a bilinear version of the estimate (17) which is closely related to bilinear restriction estimate for the cone ( [13, 23, 25, 28]). Under an additional transversality condition, bilinear estimate gives formally better estimate than linear one by weakening Kakeya compression phenomena.

Definition 2.1. For a function $f$ which is supported away from the origin we define the angular support $\mathcal{A}$ supp $f$ by

$$
\mathcal{A} \text { supp } f=\left\{\frac{\xi}{|\xi|}: \xi \in \operatorname{supp} f\right\} .
$$


The following may be regarded as a generalization of bilinear restriction estimate for the cone in [28].

Theorem 2.2. Let $R \gg 1$ and let $\mu$ be an $\alpha$-dimensional measure supported in $\overline{B(0,1)}$. Suppose that $f$ and $g$ are supported in $\Gamma_{R}(1)$ and

$$
\operatorname{dist}(\mathcal{A} \operatorname{supp} f, \mathcal{A} \operatorname{supp} g) \geq \frac{1}{100} .
$$

For $2 \leq q \leq \infty$, there is a constant $C=C(\beta, n)$ such that

$$
\left(\int|\widehat{f} \widehat{g}|^{\frac{q}{2}} d \mu\right)^{\frac{2}{q}} \leq C R^{2 \beta}\langle\mu\rangle_{\alpha}^{\frac{2}{q}}\|f\|_{2}\|g\|_{2}
$$

for any $\beta>\beta(\alpha, q):=\max \left\{\frac{n}{2}-\frac{\alpha}{q}, \frac{3 n+1-2 \alpha}{8}\right\}$.

Since $|\widehat{f}| \lesssim R^{\frac{n}{2}}\|f\|_{2}$ and $|\widehat{g}| \lesssim R^{\frac{n}{2}}\|g\|_{2}$, (10) trivially holds with $2 \beta \geq n$. It is easy to verify that the condition $\beta \geq \frac{n}{2}-\frac{\alpha}{q}$ is necessary by adopting the examples which are used to show the necessity of the condition (28). Another necessary condition for (10) with $\alpha>2$ is

$$
\beta \geq \frac{n-1}{4}-\frac{\alpha-2}{2 q} .
$$

To see this, we consider the squashed cap function (see [25]) and a suitable measure. Precisely, let us set $d \mu=\psi(x)\left|x^{\prime \prime}\right|^{-n-1+\alpha} d x_{1} d x_{2} d x^{\prime \prime}, x=\left(x_{1}, x_{2}, x^{\prime \prime}\right)$ for a smooth cutoff function $\psi$. Then $\mu$ is an $\alpha$-dimensional measure if $n+1 \geq \alpha>2$. Considering a pair of characteristic functions $f, g$ supported in $\Gamma_{R}(1)$ with large angular separation and dimensions $1 \times 1 \times \sqrt{R} \times \cdots \times \sqrt{R}$ it is easy to show (11).

Localization to a smaller cube. Improvement due to localization is important for the induction on scale argument. In the following lemma we make it precise how localization to smaller cubes affects the estimate (10).

Lemma 2.3. Let $\beta \geq 0, q \geq 2$. Suppose that (10) is valid for any $\alpha$-dimensional measure $\mu$ supported in $\overline{B(0,1)}$, and for any $f, g$ supported in $\Gamma_{R}(A)$ for some $A \sim 1$ satisfying the condition (9). Then, for $x_{0} \in \mathbb{R}^{n+1}$ and $\rho \leq(10 A)^{-1}$,

$$
\left(\int_{B\left(x_{0}, \rho\right)}|\widehat{f} \widehat{g}|^{\frac{q}{2}} d \mu\right)^{\frac{2}{q}} \leq C\langle\mu\rangle_{\alpha}^{\frac{2}{q}} R^{2 \beta} \rho^{\frac{2 \alpha}{q}+2 \beta-n}\|f\|_{2}\|g\|_{2} .
$$

Proof. By translation we may assume $x_{0}=0$ without loss of generality.

Since the map $\phi \mapsto \rho^{-\alpha} \int \phi\left(\rho^{-1} x\right) d \mu(x)$ is a positive linear functional on $C_{c}\left(\mathbb{R}^{n+1}\right)$, by the Riesz representation theorem there exists a Radon measure $\mu_{\rho}$ such that

$$
\int \phi(y) d \mu_{\rho}(y)=\rho^{-\alpha} \int \phi\left(\rho^{-1} x\right) \chi_{B(0, \rho)}(x) d \mu(x)
$$


for any continuous function $\phi$. Then it is obvious that $\mu_{\rho}$ is supported in $\overline{B(0,1)}$ and $\left\langle\mu_{\rho}\right\rangle_{\alpha} \leq\langle\mu\rangle_{\alpha}$. Let us set $f_{\rho}=\rho^{-n-1} f(\cdot / \rho), g_{\rho}=\rho^{-n-1} g(\cdot / \rho)$. Then, it follows that

$$
\int_{B(0, \rho)}|\widehat{f} \widehat{g}|^{\frac{q}{2}} d \mu=\rho^{\alpha} \int_{B(0,1)}\left|\widehat{f}_{\rho} \widehat{g}_{\rho}\right|^{\frac{q}{2}} d \mu_{\rho} .
$$

We note that $f_{\rho}$ and $g_{\rho}$ are supported in the set $\Gamma_{R \rho}(A \rho)$. Since the measure $\mu_{\rho}$ is supported in $\overline{B(0,1)}$, we may put a harmless smooth function $\eta$ in the integral which satisfies $\widehat{\eta} \sim 1$ on $\overline{B(0,1)}$ and $\operatorname{supp} \eta \subset B\left(0, \frac{1}{2}\right)$. Then we see

$$
\begin{aligned}
\int\left|\widehat{f}_{\rho}(x) \widehat{g}_{\rho}(x)\right|^{\frac{q}{2}} d \mu_{\rho}(x) & \sim \int\left|\widehat{\eta}^{2} \widehat{f}_{\rho}(x) \widehat{g}_{\rho}(x)\right|^{\frac{q}{2}} d \mu_{\rho}(x) \\
& =\left.\int \widehat{\mid \eta * f_{\rho}}(x) \widehat{\eta * g_{\rho}}(x)\right|^{\frac{q}{2}} d \mu_{\rho}(x) .
\end{aligned}
$$

Note that $\eta * f_{\rho}$ and $\eta * g_{\rho}$ are supported in $\Gamma_{R \rho}(1)$. Hence, from (10) we have

$$
\left(\left.\int \widehat{\mid \eta * f_{\rho}}(x) \widehat{\eta * g_{\rho}}(x)\right|^{\frac{q}{2}} d \mu_{\rho}(x)\right)^{\frac{2}{q}} \leq C\langle\mu\rangle_{\alpha}^{\frac{2}{q}}(R \rho)^{2 \beta}\left\|\eta * f_{\rho}\right\|_{2}\left\|\eta * g_{\rho}\right\|_{2} .
$$

Since $f_{\rho}$ and $g_{\rho}$ are supported in $\Gamma_{R \rho}(A \rho),\left\|\eta * f_{\rho}\right\|_{2} \leq C \rho^{-n / 2}\|f\|_{2}$ i) and $\left\|\eta * g_{\rho}\right\|_{2} \leq$ $C \rho^{-n / 2}\|g\|_{2}$. Therefore (12) follows by (13) and (14).

Decomposition of $f$ and $g$. For the proof of Theorem 2.2 we adapt the induction on scale argument which has been used to prove sharp bilinear restriction estimate [13, 23, 28]. The following is actually a rescaled version of [28, Lemma 3.5] (also see [13, 23] for a simpler proof based on wave packet decomposition, especially the proof of Theorem 1.3 in [13]).

Lemma 2.4. Let $R \gg 1$ and $0<\delta \leq 1$. Let $\{\mathbf{q}\}$ be a collection of cubes of sidelength $\sim R^{-\delta}$ which partition the cube $[-1,1]^{n+1}$. Suppose $f$ and $g$ are supported in $\Gamma_{R}(1)$ and satisfy (9). Then for each $\mathbf{q}, f$ and $g$ can be decomposed such that

$$
f=f_{\mathbf{q}}+f_{\underset{\mathbf{q}}{x}} \text { and } g=g_{\mathbf{q}}+g_{\nsim}
$$

with $f_{\mathbf{q}}, f_{\underset{\mathbf{q}}{\chi}}, g_{\mathbf{q}}, g_{\underset{\mathbf{q}}{x}}$ supported in $\Gamma_{R}\left(C_{d}\right)$ for some $C_{d} \sim 1$ and, for $0<\epsilon \ll \delta$,

$$
\begin{aligned}
& \sum_{\mathbf{q}}\left\|f_{\mathbf{q}}\right\|_{2}^{2} \leq C R^{\epsilon}\|f\|_{2}^{2}, \quad \sum_{\mathbf{q}}\left\|g_{\mathbf{q}}\right\|_{2}^{2} \leq C R^{\epsilon}\|g\|_{2}^{2}, \\
& \left\|\widehat{f_{\mathbf{q}}} \widehat{g_{\mathbf{q}}}\right\|_{L^{2}(\mathbf{q})}, \quad\left\|\widehat{f_{\chi}} \widehat{g_{\mathbf{q}}}\right\|_{L^{2}(\mathbf{q})}, \quad\left\|\widehat{f_{\mathbf{q}^{\prime}}} \widehat{\hat{q}_{\mathbf{q}}}\right\|_{L^{2}(\mathbf{q})} \leq C R^{\frac{n-1}{4}+c \delta+\epsilon}\|f\|_{2}\|g\|_{2}
\end{aligned}
$$

with $c$ independent of $\delta$ and $\epsilon$.

\footnotetext{
i) This follows from Hölder inequality and easy estimates $\left\|\eta * f_{\rho}\right\|_{1} \lesssim\|f\|_{1},\left\|\eta * f_{\rho}\right\|_{\infty} \lesssim \rho^{-n}\|f\|_{\infty}$.
} 
Proof. Let $F$ and $G$ be functions supported in $\Gamma_{1}\left(R^{-1}\right)$. Then, by using wave packet decompositionii) ( $[13,23])$, on $B(0, R)$ we may write

$$
\widehat{F}=\sum_{w_{1}} c_{w_{1}} p_{w_{1}} \quad \text { and } \quad \widehat{G}=\sum_{w_{2}} c_{w_{2}} p_{w_{2}}
$$

where $c_{w_{i}}$ and $p_{w_{i}}$ satisfy the following:

(1) $\left(\sum_{w_{1} \in W_{1}}\left|c_{w_{1}}\right|^{2}\right)^{1 / 2} \leq C\|F\|_{2}, \quad\left(\sum_{w_{2} \in W_{2}}\left|c_{w_{2}}\right|^{2}\right)^{1 / 2} \leq C\|G\|_{2}$

(2) If $w_{i} \in W_{i}$, supp $\widehat{p_{w_{i}}} \subset\left\{\xi: \xi=v_{i}+O\left(R^{-1 / 2}\right)\right\}$;

(3) If $\operatorname{dist}\left(x, T_{w_{i}}\right) \geq R^{1 / 2+\delta},\left|p_{w_{i}}(x)\right| \leq C R^{-100 n}$;

(4) For any subset $S \subset W_{i},\left\|\sum_{w_{i} \in S} p_{w_{i}}\right\|_{2}^{2} \leq C \# S$.

Here $W_{i}$ is the set of all pairs $w_{i}=\left(y_{i}, v_{i}\right)$ with $y_{i} \in R^{1 / 2} \mathbb{Z}^{n}$ and $v_{i} \in R^{-1 / 2} \mathbb{Z}^{n} \cap\{v \in$ $\left.\mathbb{R}^{n}: 2^{-1} \leq|v| \leq 2^{2}\right\}$ ) and $T_{w_{i}}$ is defined by

$$
T_{w_{i}}=\left\{\left(x^{\prime}, x_{n+1}\right) \in \mathbb{R}^{n} \times \mathbb{R}:\left|x_{n+1}\right| \leq R,\left|x^{\prime}-\left(y_{i}+x_{n+1} \frac{v_{i}}{\left|v_{i}\right|}\right)\right| \leq R^{1 / 2}\right\}
$$

for each $w_{i} \in W_{i}, i=1,2$.

Partition $[-R, R]^{n+1}$ into $R^{1-\delta}$-cubes $\{R \mathbf{q}\}$ which are essentially disjoint. Then,

$$
\|\widehat{F} \widehat{G}\|_{L^{q}(B(0, R))} \leq \sum_{\mathbf{q}}\|\widehat{F} \widehat{G}\|_{L^{q}(R \mathbf{q})}
$$

Now we use the relation $\sim$ between $w_{i}$ and $R^{1-\delta}$ cube $R \mathbf{q}$ which was introduced in [23] (also see [13]). For $\mathbf{q}$ fixed, we say $w_{i} \sim \mathbf{q}$ if $w_{i} \sim R \mathbf{q}$ and, otherwise, we say $w_{i} \not \mathbf{q}$. We keep the same notation $\sim$ since it does not cause any ambiguity. We now set

$$
\widehat{F_{\mathbf{q}}}=\sum_{w_{1} \sim \mathbf{q}} c_{w_{1}} p_{w_{1}}, \quad \widehat{F_{\ngtr}}=\sum_{w_{1} \not \mathbf{q}} c_{w_{1}} p_{w_{1}}, \quad \widehat{G_{\mathbf{q}}}=\sum_{w_{2} \sim \mathbf{q}} c_{w_{2}} p_{w_{2}} \quad \widehat{G_{\ngtr}}=\sum_{w \neq \mathbf{q}} c_{w_{2}} p_{w_{2}} .
$$

Then, by repeating the argument in [13,23] it is not difficult to see that

$$
\begin{gathered}
\sum_{\mathbf{q}}\left\|\widehat{F_{\mathbf{q}}}\right\|_{2}^{2} \lesssim R^{\epsilon}\|F\|_{2}^{2}, \quad \sum_{\mathbf{q}}\left\|\widehat{G_{\mathbf{q}}}\right\|_{2}^{2} \lesssim R^{\epsilon}\|G\|_{2}^{2} ; \\
\left\|\widehat{F_{\mathbf{q}}} \widehat{G_{\mathbf{q}}}\right\|_{L^{2}(R \mathbf{q})}, \quad\left\|\widehat{F_{\ngtr}} \widehat{G_{\mathbf{q}}}\right\|_{L^{2}(R \mathbf{q})}, \quad\left\|\widehat{F_{\underset{\mathbf{q}}{*}}} \widehat{G_{\boldsymbol{q}}}\right\|_{L^{2}(R \mathbf{q})} \lesssim R^{-\frac{n+3}{4}+c \delta+\epsilon}\|F\|_{2}\|G\|_{2} .
\end{gathered}
$$

Since we are dealing with functions $F$ and $G$ which are supported in $\Gamma_{1}\left(R^{-1}\right)$ instead of being supported on the surfaces, we obtain an extra power $R^{-1}$ compared to the extension operator ( $c f$. [13, 23]).

\footnotetext{
ii) It should be noted that we are working with functions instead of extension operators. Nevertheless, the argument in [13] works without extra difficulty.
} 
Since $f, g$ are supported in $\Gamma_{R}(1)$ and satisfy (9), we apply the above to

$$
F=R^{\frac{n+1}{2}} f(R \cdot) \quad \text { and } \quad G=R^{\frac{n+1}{2}} g(R \cdot) .
$$

Then, the change of variable $x \rightarrow x / R$ gives the desired estimates (15) and (16).

To prove Theorem 2.2 we make use of the following simple lemma.

Lemma 2.5. Let $R \gg 1$ and $\mu$ be an $\alpha$-dimensional measure supported in $\overline{B(0,1)}$. Set $\phi_{R}=R^{n+1} \phi(R \cdot)$ for a Schwartz function $\phi$. Then, for any $r \geq 1$ and any Schwartz function $\phi$, there is a constant $C$, independent of $\mu$, such that

$$
\left\|\phi_{R} * d \mu\right\|_{r} \leq C\langle\mu\rangle_{\alpha} R^{(n+1-\alpha)\left(1-\frac{1}{r}\right)} .
$$

Proof. By rapid decay of $\phi$ it follows that $\phi_{R} \leq C_{N} R^{n+1} \sum_{j \geq 0} 2^{-N j} \chi_{B\left(0,2^{j} R^{-1}\right)}$ for all $N>0$. Hence, recalling the definition of $\langle\mu\rangle_{\alpha}$ in Definition 1.1 and choosing a sufficiently large $N$, we have

$$
\begin{aligned}
\phi_{R} * d \mu(x) & \leq C_{N} R^{n+1} \sum_{j \geq 0} 2^{-N j} \mu\left(B\left(x, 2^{j} R^{-1}\right)\right) \\
& \leq C_{N}\langle\mu\rangle_{\alpha} R^{n+1-\alpha} \sum_{j \geq 0} 2^{(\alpha-N) j} \leq C_{N}\langle\mu\rangle_{\alpha} R^{n+1-\alpha} .
\end{aligned}
$$

This yields (17) with $r=\infty$. Since $\mu$ is supported in $\overline{B(0,1)}$, we also have $\|\mu\| \leq$ $2^{\alpha}\langle\mu\rangle_{\alpha}$ where $\|\mu\|=\sup \left\{\left|\int f(x) d \mu(x)\right|:|f| \leq 1, f \in C\left(\mathbb{R}^{n+1}\right)\right\}$. So, from Young inequality $\left\|\phi_{R} * d \mu\right\|_{1} \leq\left\|\phi_{R}\right\|_{1}\|\mu\| \lesssim\langle\mu\rangle_{\alpha}$. Hence, (17) follows from $\left\|\phi_{R} * d \mu\right\|_{r}^{r} \leq$ $\left\|\phi_{R} * d \mu\right\|_{\infty}^{r-1}\left\|\phi_{R} * d \mu\right\|_{1}$ for any $r \geq 1$.

Proof of Theorem 2.2. It is sufficient to show the case $2 \leq q \leq 4$. Extension to $q \geq 4$ can be obtained by interpolation with the trivial estimate

$$
\|\widehat{f} \widehat{g}\|_{L^{\infty}(d \mu)} \lesssim R^{n}\|f\|_{L^{2}}\|g\|_{L^{2}}
$$

which is valid since $\widehat{f}$ and $\widehat{g}$ are continuous.

In what follows we prove the implication from (10) to (20). We assume that (10) holds for some $\beta>0$ and $f, g$ supported in $\Gamma_{R}(1)$. As is mentioned before, this is true with a large $\beta>0$. Since $\mu$ is supported in $\overline{B(0,1)}$, we have

$$
\left(\int|\widehat{f} \widehat{g}|^{q / 2} d \mu\right)^{\frac{2}{q}} \leq \sum_{\mathbf{q}}\left(\int_{\mathbf{q}}|\widehat{f} \widehat{g}|^{q / 2} d \mu\right)^{\frac{2}{q}}
$$

Using the decomposition in Lemma 2.4, we have

$$
\begin{aligned}
& \left(\int|\widehat{f} \widehat{g}|^{q / 2} d \mu\right)^{\frac{2}{q}} \leq \sum_{\mathbf{q}}\left[\left(\int_{\mathbf{q}}\left|\widehat{f}_{\mathbf{q}} \widehat{g}_{\mathbf{q}}\right|^{q / 2} d \mu\right)^{\frac{2}{q}}+\left(\int_{\mathbf{q}}\left|\widehat{f}_{\mathbf{q}}{\widehat{g_{\mathbf{q}}}}_{\mathbf{q}}\right|^{q / 2} d \mu\right)^{\frac{2}{q}}\right. \\
& \left.+\left(\int_{\mathbf{q}}\left|\widehat{f_{\prec}} \widehat{g_{\mathbf{q}}}\right|^{q / 2} d \mu\right)^{\frac{2}{q}}+\left(\int_{\mathbf{q}}\left|\widehat{f_{\ngtr}} \widehat{g_{\mathcal{q}}}\right|^{q / 2} d \mu\right)^{\frac{2}{q}}\right] \text {. }
\end{aligned}
$$


By Lemma 2.3, it follows that

$$
\left(\int_{\mathbf{q}}\left|\widehat{f}_{\mathbf{q}} \widehat{g}_{\mathbf{q}}\right|^{q / 2} d \mu\right)^{\frac{2}{q}} \leq C\langle\mu\rangle_{\alpha}^{\frac{2}{q}} R^{2 \beta} R^{\delta\left(n-\frac{2 \alpha}{q}-2 \beta\right)}\left\|f_{\mathbf{q}}\right\|_{2}\left\|g_{\mathbf{q}}\right\|_{2} .
$$

Schwarz inequality and (15) give

$$
\sum_{\mathbf{q}}\left(\int_{\mathbf{q}}\left|\widehat{f}_{\mathbf{q}} \widehat{g}_{\mathbf{q}}\right|^{q / 2} d \mu\right)^{\frac{2}{q}} \lesssim C\langle\mu\rangle_{\alpha}^{\frac{2}{q}} R^{2 \beta+\epsilon} R^{\delta\left(n-\frac{2 \alpha}{q}-2 \beta\right)}\|f\|_{2}\|g\|_{2} .
$$

For the other terms in (18) we use (16). Let $\eta$ be a Schwartz function such that $\widehat{\eta}(\xi)=1$ if $|\xi| \leq 10 C$ and $\operatorname{supp} \widehat{\eta} \subset B(0,20 C)$ and set $\eta_{R}=R^{n+1} \eta(R \cdot)$. Since $f_{\mathbf{q}} * g_{\Varangle}$ is supported in a ball of radius $10 R$, we have $\left|\widehat{f_{\mathbf{q}}} \widehat{\widehat{g}_{\mathbf{q}}}\right|=\left|\left(\widehat{f_{\mathbf{q}}} \widehat{g_{\mathbf{q}}}\right) * \eta_{R}\right| \lesssim\left(\left|{\widehat{f_{\mathbf{q}}}}_{\mathrm{g}_{\mathbf{q}}}\right|^{q / 2} * \eta_{R}\right)^{2 / q}$. By (17), we thus obtain

$$
\begin{aligned}
\left(\int_{\mathbf{q}}\left|\eta_{R} *\left(\widehat{f_{\mathbf{q}}} \widehat{g_{\mathbf{q}}}\right)\right|^{q / 2} d \mu\right)^{\frac{2}{q}} & \leq C\left(\int_{\mathbf{q}}\left|\left(\widehat{f_{\mathbf{q}}} \widehat{g_{\mathbf{q}}}\right)\right|^{q / 2}\left|\eta_{R}\right| * d \mu\right)^{\frac{2}{q}} \\
& \leq C \begin{cases}\left\|\widehat{f_{\mathbf{q}}} \widehat{g_{\prec}}\right\|_{L^{2}(\mathbf{q})}\left\|\left|\eta_{R}\right| * d \mu\right\|_{\infty}^{\frac{1}{2}} & \text { if } q=4 \\
\left\|\widehat{f_{\mathbf{q}}} \widehat{g_{\mathbf{q}}}\right\|_{L^{2}(\mathbf{q})}\left\|\left|\eta_{R}\right| * d \mu\right\|_{2} & \text { if } q=2 .\end{cases}
\end{aligned}
$$

By (16) it follows that for $2 \leq q \leq 4$,

$$
\begin{aligned}
\left(\int_{\mathbf{q}}\left|\widehat{f}_{\mathbf{q}}{\widehat{g_{\chi}}}_{\mathbf{q}}\right|^{q / 2} d \mu\right)^{\frac{2}{q}} & \leq C\left\|\widehat{f}_{\mathbf{q}} \widehat{g}_{\mathbf{q}}\right\|_{L^{2}(\mathbf{q})}\langle\mu\rangle_{\alpha}^{\frac{2}{q}} R^{\frac{n+1-\alpha}{2}} \\
& \leq C\langle\mu\rangle_{\alpha}^{\frac{2}{q}} R^{\frac{3 n+1-2 \alpha}{4}+c \delta+\epsilon}\|f\|_{2}\|g\|_{2} .
\end{aligned}
$$

The terms $\widehat{f_{\chi_{\mathbf{q}}}} \widehat{g_{\mathbf{q}}}$ and $\widehat{f_{\chi_{\mathbf{q}}}} \widehat{g_{\mathbf{q}}}$ can be treated similarly. Since $\#\{\mathbf{q}\} \leq C R^{(n+1) \delta}$, combining the estimates for these terms, we get

$$
\begin{aligned}
\sum_{\mathbf{q}}\left(\int_{\mathbf{q}}\left|\widehat{f}_{\mathbf{q}} \widehat{g_{\mathbf{q}}}\right|^{q / 2} d \mu\right)^{\frac{2}{q}} & +\left(\int_{\mathbf{q}}\left|\widehat{f_{\not}} \widehat{g_{\mathbf{q}}}\right|^{q / 2} d \mu\right)^{\frac{2}{q}}+\left(\int_{\mathbf{q}}\left|\widehat{f_{\ngtr}} \widehat{g_{\mathbf{q}}}\right|_{\mathbf{q}}^{q / 2} d \mu\right)^{\frac{2}{q}} \\
& \leq C\langle\mu\rangle_{\alpha}^{\frac{2}{q}} R^{\frac{3 n+1-2 \alpha}{4}+\tilde{c} \delta+\epsilon}\|f\|_{2}\|g\|_{2} .
\end{aligned}
$$

Here $\tilde{c}=c+(n+1)$. By this and (19) it follows from (18) that for any $\epsilon>0$,

$$
\left(\int|\widehat{f} \widehat{g}|^{q / 2} d \mu\right)^{\frac{2}{q}} \leq C\langle\mu\rangle_{\alpha}^{\frac{2}{q}} R^{\max \left\{2 \beta+\delta\left(n-\frac{2 \alpha}{q}-2 \beta\right), \frac{3 n+1-2 \alpha}{4}+\tilde{c} \delta\right\}+\epsilon}\|f\|_{2}\|g\|_{2} \text {. }
$$

Hence we have shown that (10) implies (20). If we have (10) for $\beta=\beta_{i}$, then we see that (10) holds with

$$
\beta=\frac{3 n+1-2 \alpha}{8}+\tilde{c}\left(\frac{8 \beta_{i}-3 n-1+2 \alpha}{8\left(\tilde{c}-n+2 \alpha / q+2 \beta_{i}\right)}\right):=\beta_{i+1}
$$

by choosing $\delta=\frac{8 \beta-3 n-1+2 \alpha}{4(\tilde{c}-n+2 \alpha / q+2 \beta)}$. Iterating this implication we obtain a sequence $\left\{\beta_{i}\right\}_{i=0}^{\infty}$. Note that the sequence $\left\{\beta_{i}\right\}_{i=0}^{\infty}$ is strictly decreasing as long as $-n+2 \alpha / q+$ 
$2 \beta_{i}>0$ i.e. $\beta_{i}>n / 2-\alpha / q$. Since $\beta_{i}$ converges to $(3 n+1-2 \alpha) / 8$, we conclude that (10) holds for $2 \leq q \leq 4$ and for any $\beta>\max \left\{\frac{n}{2}-\frac{\alpha}{q}, \frac{3 n+1-2 \alpha}{8}\right\}$. This completes the proof.

\section{Proof of Theorem 1.3: Rescaling}

In this section we prove the following theorem by deducing linear estimate from the bilinear one (10).

Theorem 3.1. Let $n \geq 3$ and $\mu$ be an $\alpha$-dimensional measure supported in $\overline{B(0,1)}$. Then, for $f$ supported in $\Gamma_{R}(1)$ there exists a constant $C>0$ such that (7) holds for $s$ satisfying

$$
s>\widetilde{s}(\alpha, q, d):= \begin{cases}\max \left\{\frac{n}{2}-\frac{\alpha}{q}, \frac{n+1}{4}, \frac{3 n+1}{8}-\frac{\alpha}{4}\right\}, & \text { if } 0<\alpha \leq 1, \\ \max \left\{\frac{n}{2}-\frac{\alpha}{q}, \frac{n+1}{4}+\frac{1-\alpha}{2 q}, \frac{3 n+1}{8}-\frac{\alpha}{4}\right\}, & \text { if } 1<\alpha \leq n, \\ \max \left\{\frac{n}{2}-\frac{\alpha}{q}, \frac{n+1}{4}+\frac{n+1-2 \alpha}{2 q}, \frac{n+1-\alpha}{2}\right\}, & \text { if } n<\alpha \leq n+1 .\end{cases}
$$

For $n=3$, this immediately implies Theorem 1.3 .

Proof of Theorem 1.3. It suffices to check that $\widetilde{s}(\alpha, q, 3)=s(\alpha, q, 3)$ for $0<\alpha \leq 3$. If $0<\alpha \leq 1$, it is easy to check $\widetilde{s}(\alpha, q, 3)=n / 2-\alpha / q=s(\alpha, q, 3)$ for $q \geq 2$. If $1<\alpha \leq 3$, we see that $\widetilde{s}(\alpha, q, 3)=s(\alpha, q, 3)$ because $(3 n+1-2 \alpha) / 8=(n+2-\alpha) / 4$ when $n=3$. This completes the proof.

To prove Theorem 3.1, we begin with a Whitney type decomposition to exploit bilinear estimate. (See [25, 28].)

Whitney type decomposition of $\mathbb{S}^{n-1} \times \mathbb{S}^{n-1}$. For each $j \geq 1$, let us dyadically divide the sphere $\mathbb{S}^{n-1}$ into $O\left(2^{(n-1) j}\right)$ caps $\theta_{k}^{j}$ of diameter $\sim 2^{-j}$. We will write $\theta_{k}^{j} \approx \theta_{k^{\prime}}^{j}$ to mean that $\theta_{k}^{j}$ and $\theta_{k^{\prime}}^{j}$ are not adjacent but have adjacent parent caps of diameter $\sim 2^{1-j}$. Then, by Whitney decomposition of $\mathbb{S}^{n-1} \times \mathbb{S}^{n-1}$ away from its diagonal $D=\left\{(x, x): x \in \mathbb{S}^{n-1}\right\}$ we have

$$
\mathbb{S}^{n-1} \times \mathbb{S}^{n-1} \backslash D=\bigcup_{j \geq 1} \bigcup_{\left(k, k^{\prime}\right): \theta_{k}^{j} \approx \theta_{k^{\prime}}^{j}} \theta_{k}^{j} \times \theta_{k^{\prime}}^{j}
$$

Let $D\left(R^{-1 / 2}\right)$ be a $O\left(R^{-1 / 2}\right)$ neighborhood of $D$. Then $\mathbb{S}^{n-1} \times \mathbb{S}^{n-1} \backslash D\left(R^{-1 / 2}\right)$ can be covered by $\theta_{k}^{j} \times \theta_{k^{\prime}}^{j}$ such that $\theta_{k}^{j} \approx \theta_{k^{\prime}}^{j}$ and $1 \leq j \leq \log R^{1 / 2}$. Also $D\left(R^{-1 / 2}\right)$ is covered by a union of disjoint cubes $\theta_{k}^{j_{\circ}} \times \theta_{k}^{j_{\circ}}$ of sidelength $O\left(R^{-1 / 2}\right)$. So, $2^{-j_{\circ}} \sim 1 / \sqrt{R}$.

For $f$ supported away from the origin we set

$$
f_{k}^{j}(\xi, \tau)=\chi_{\theta_{k}^{j}}(\xi / \tau) f(\xi, \tau), \quad(\xi, \tau) \in \mathbb{R}^{n} \times \mathbb{R} .
$$

Then by the above decomposition we have

$$
|\widehat{f}|^{2} \leq \sum_{j=1}^{\log R^{1 / 2}} \sum_{\left(k, k^{\prime}\right): \theta_{k}^{j} \approx \theta_{k^{\prime}}^{j}}\left|\widehat{f_{k}^{j}} \widehat{f_{k^{\prime}}^{j}}\right|+\sum_{k}\left|\widehat{f_{k}^{j_{\circ}}}\right|^{2} .
$$


Note that the diameters of $\mathcal{A}$ supp $f_{k}^{j}$ and $\mathcal{A}$ supp $f_{k^{\prime}}^{j}$ are $O\left(2^{-j}\right)$. In order to handle the first sum we need the following.

Lemma 3.2. Let $1 \leq j \leq \log \left(R^{1 / 2}\right)$. Suppose $f$ and $g$ are supported in $\Gamma_{R}(1)$ and the diameters of $\mathcal{A}$ supp $f$ and $\mathcal{A}$ supp $g \leq 2^{-j-2}$. If $\operatorname{dist}(\mathcal{A}$ supp $f, \mathcal{A}$ suppg $) \geq 2^{-j}$, there exists a constant $C>0$ such that

$$
\|\widehat{f} \widehat{g}\|_{L^{\frac{q}{2}(d \mu)}} \leq C\langle\mu\rangle_{\alpha}^{\frac{2}{q}} R^{2 \beta} 2^{\gamma j}\|f\|_{2}\|g\|_{2},
$$

where $\beta>\beta(\alpha, q)$ and

$$
\gamma=\gamma(\alpha, \beta, n)= \begin{cases}-4 \beta+2(n+1-2 \alpha) / q+n+1, & \text { if } n<\alpha \leq n+1, \\ -4 \beta+2(1-\alpha) / q+n+1, & \text { if } 1 \leq \alpha \leq n, \\ -4 \beta+n+1, & \text { if } 0<\alpha \leq 1\end{cases}
$$

Proof. By rotation we may assume that $\mathcal{A}$ supp $f$ and $\mathcal{A}$ suppg are contained in $O\left(2^{-j}\right)$ neighborhood of $\left(0, \ldots, 0, \frac{1}{\sqrt{2}}, \frac{1}{\sqrt{2}}\right) \in \mathbb{R}^{n+1}$. More precisely, we use the null coordinates

$$
\xi^{\prime \prime}=\left(\xi_{1}, \xi_{2}, \ldots, \xi_{n-1}\right), \sigma=\frac{\xi_{n+1}-\xi_{n}}{\sqrt{2}}, \tau=\frac{\xi_{n+1}+\xi_{n}}{\sqrt{2}} .
$$

Then the cone $\Gamma_{R}$ can be written as $\left|\xi^{\prime \prime}\right|^{2}=2 \sigma \tau$. So

$$
\Gamma_{R}(1) \subset\left\{\left(\xi^{\prime \prime}, \sigma, \tau\right):\left|\xi^{\prime \prime}\right|^{2}=2 \sigma \tau+O(1), \tau \sim R\right\} .
$$

Additionally, after a slight adjustment we may assume that supp $f \subset V_{1}$ and $\operatorname{supp} g \subset$ $V_{2}$, where

$V_{i}=\left\{\left(\xi^{\prime \prime}, \sigma, \tau\right):\left|\xi^{\prime \prime}\right|^{2}=2 \sigma \tau+O(1), \tau \sim R,\left|\xi^{\prime \prime} / \tau+(-1)^{i} 2^{-j} e_{1}\right| \leq 2^{-j-2}\right\}, i=1,2$.

Now, using an anisotropic transformation $T_{j}:\left(\xi^{\prime \prime}, \sigma, \tau\right) \mapsto\left(2^{j} \xi^{\prime \prime}, \sigma, 2^{2 j} \tau\right)$, we have

$$
\|\widehat{f} \widehat{g}\|_{L^{q / 2}(d \mu)}=\left\|\widehat{f}_{j} \widehat{g}_{j}\right\|_{L^{q / 2}\left(d \mu_{j}\right)},
$$

where $f_{j}=\left|\operatorname{det} T_{j}\right| f \circ T_{j}=2^{(n+1) j} f \circ T_{j}, g_{j}=\left|\operatorname{det} T_{j}\right| g \circ T_{j}$, and

$$
\mu_{j}(\phi)=\int \phi \circ T_{j} d \mu \text {. }
$$

So, we see

$$
\operatorname{supp} f_{j} \subset\left\{\left(\xi^{\prime \prime}, \sigma, \tau\right):\left|\xi^{\prime \prime}\right|^{2}=2 \sigma \tau+O\left(2^{-2 j}\right), \tau \sim 2^{-2 j} R,\left|\xi^{\prime \prime} / \tau-e_{1}\right| \leq 2^{-2}\right\}
$$

and

$$
\operatorname{supp} g_{j} \subset\left\{\left(\xi^{\prime \prime}, \sigma, \tau\right):\left|\xi^{\prime \prime}\right|^{2}=2 \sigma \tau+O\left(2^{-2 j}\right), \tau \sim 2^{-2 j} R,\left|\xi^{\prime \prime} / \tau+e_{1}\right| \leq 2^{-2}\right\} .
$$

Clearly, $\mu_{j}$ is supported in $\overline{B(0,1)}$ because $j \geq 1$, and note that $f$ and $g$ are supported in $\Gamma_{2^{-2 j} R}\left(2^{-2 j}\right)$ and satisfy the separation condition (9) in Theorem 2.2. by which we have that, for $\beta>\beta(\alpha, q)$,

$$
\left\|\widehat{f}_{j} \widehat{g}_{j}\right\|_{L^{q / 2}\left(d \mu_{j}\right)} \leq C\left\langle\mu_{j}\right\rangle_{\alpha}^{\frac{2}{q}}\left(2^{-2 j} R\right)^{2 \beta}\left\|f_{j}\right\|_{2}\left\|g_{j}\right\|_{2} .
$$


We now estimate $\left\langle\mu_{j}\right\rangle_{\alpha}$. For any $(x, \rho) \in \mathbb{R}^{n+1} \times \mathbb{R}$, we have

$$
\mu_{j}(B(x, \rho))=\int_{T_{j}^{-1} B(x, \rho)} d \mu \lesssim \int_{\mathcal{R}} d \mu
$$

where $\mathcal{R}$ is a rectangle of dimensions $2^{-j} \rho \times 2^{-j} \rho \times \cdots \times 2^{-j} \rho \times \rho \times 2^{-2 j} \rho$. There are three different ways of decomposing $\mathcal{R}$ into cubes, namely, cubes of side length $2^{-2 j} \rho, 2^{-j} \rho$, and $\rho$, respectively. Considering these three cases and using (4) for each case, we obtain

$$
\begin{aligned}
& \mu_{j}(B(x, \rho)) \lesssim\langle\mu\rangle_{\alpha} \min \left\{2^{(n+1-2 \alpha) j} \rho^{\alpha}, 2^{(1-\alpha) j} \rho^{\alpha}, \rho^{\alpha}\right\} \\
&=\langle\mu\rangle_{\alpha} \times \begin{cases}2^{(n+1-2 \alpha) j} \rho^{\alpha}, & \text { if } n<\alpha \leq n+1, \\
2^{(1-\alpha) j} \rho^{\alpha}, & \text { if } 1<\alpha \leq n, \\
\rho^{\alpha}, & \text { if } 0<\alpha \leq 1 .\end{cases}
\end{aligned}
$$

Hence, $\left\langle\mu_{j}\right\rangle_{\alpha} \lesssim \min \left\{2^{(n+1-2 \alpha) j}, 2^{(1-\alpha) j}, 1\right\} \times\langle\mu\rangle_{\alpha}$. Combining this with (23),$\left\|f_{j}\right\|_{2}=$ $2^{j(n+1) / 2}\|f\|_{2}$ and $\left\|g_{j}\right\|_{2}=2^{j(n+1) / 2}\|g\|_{2}$ yields (22) .

To handle the second sum in (21), which is easier, we use the following.

Lemma 3.3. Suppose that $f$ is supported in $\Gamma_{R}(1)$ and the diameter of Asupp $f$ is $O\left(R^{-1 / 2}\right)$. For $q \geq 2$, there exists a constant $C>0$ such that

$$
\|\widehat{f}\|_{L^{q}(d \mu)} \leq C\langle\mu\rangle_{\alpha}^{\frac{1}{q}} R^{\beta_{\circ}(\alpha, q)}\|f\|_{2}
$$

where

$$
\beta_{\circ}(\alpha, q)= \begin{cases}(n+1) / 4+(n+1-2 \alpha) / 2 q, & \text { if } n<\alpha \leq n+1, \\ (n+1) / 4+(1-\alpha) / 2 q, & \text { if } 1 \leq \alpha \leq n, \\ (n+1) / 4, & \text { if } 0<\alpha \leq 1 .\end{cases}
$$

Proof. Note that $f$ is supported in a rectangle $\mathcal{R}$ of dimensions $C R^{1 / 2} \times \cdots \times C R^{1 / 2} \times$ $C \times C R$ for a positive constant $C$. We consider $T_{R}$ which takes the unit cube $Q$ onto $\mathcal{R}$. Then we have

$$
\widehat{f}(x)=R^{\frac{n+1}{2}} \int e^{-i\left(T_{R} x\right) \cdot y} f_{R}(y) d y,
$$

where $f_{R}:=f \circ T_{R}$ is supported in the unit cube $Q$.

As before, let $\mu_{R}$ be the measure given by $\mu_{R}(\phi)=\int \phi \circ T_{R} d \mu$ for continuous function $\phi$. Since $f_{R}$ is supported in $Q,\left|\widehat{f_{R}}\right| \leq C\left(\left|\widehat{f_{R}}\right|^{q} *\left|\eta_{Q}\right|\right)^{\frac{1}{q}}$ for any Schwartz function $\eta_{Q}$ satisfying $\eta_{Q}=1$ on $Q$. Using Hausdorff-Young inequality, Lemma 2.5. and Hölder inequality we have, for $q \geq 2$,

$$
\begin{gathered}
\|\widehat{f}\|_{L^{q}(d \mu)} \lesssim R^{\frac{n+1}{2}}\left\|\left|\widehat{\eta_{Q}}\right| * d \mu_{R}\right\|_{\infty}^{\frac{1}{q}}\left\|\widehat{f_{R}}\right\|_{L^{q}\left(\mathbb{R}^{n+1}\right)} \\
\lesssim R^{\frac{n+1}{2}}\left\langle\mu_{R}\right\rangle_{\alpha}^{\frac{1}{q}}\left|\operatorname{supp} f_{R}\right|^{\frac{1}{2}-\frac{1}{q}}\left\|f_{R}\right\|_{L^{2}\left(\mathbb{R}^{n+1}\right)} \lesssim R^{\frac{n+1}{4}}\left\langle\mu_{R}\right\rangle_{\alpha}^{\frac{1}{q}}\|f\|_{L^{2}\left(\mathbb{R}^{n+1}\right)}
\end{gathered}
$$


As in the proof of Theorem 3.2 (this corresponds to the case $2^{j} \sim \sqrt{R}$ ), it is easy to see

$$
\left\langle\mu_{R}\right\rangle_{\alpha}^{\frac{1}{q}} \lesssim\langle\mu\rangle_{\alpha}^{\frac{1}{q}} \times \begin{cases}R^{\frac{n+1-2 \alpha}{2 q}}, & \text { if } n<\alpha \leq n+1 \\ R^{\frac{1-\alpha}{2 q}}, & \text { if } 1<\alpha \leq n \\ 1, & \text { if } 0<\alpha \leq 1\end{cases}
$$

Therefore, combining these two estimates gives (24).

Proof of Theorem 3.1. By (21), we have

$$
\|\widehat{f}\|_{L^{q}(d \mu)}^{2} \leq \sum_{j=1}^{\log R^{1 / 2}} \sum_{\left(k, k^{\prime}\right): \theta_{k}^{j} \approx \theta_{k^{\prime}}^{j}}\left\|\widehat{f_{k}^{j}} \widehat{f_{k^{\prime}}^{j}}\right\|_{L^{q / 2}(d \mu)}+\sum_{k}\left\|\widehat{f_{k}^{j_{0}}}\right\|_{L^{q}(d \mu)}^{2}=: I+I I .
$$

By Lemma 3.2, for any $\epsilon>0$ and $R>1$, we have

$$
I \lesssim\langle\mu\rangle_{\alpha}^{\frac{2}{q}} R^{2 \beta} \sum_{j=1}^{\log R^{1 / 2}} 2^{\gamma j} \sum_{\left(k, k^{\prime}\right): \theta_{k}^{j} \approx \theta_{k^{\prime}}^{j}}\left\|f_{k}^{j}\right\|_{2}\left\|f_{k^{\prime}}^{j}\right\|_{2} .
$$

Hence, by Schwarz inequality, we get

$$
I \lesssim\langle\mu\rangle_{\alpha}^{\frac{2}{q}} R^{2 \beta} \times\left\{\begin{array}{ll}
\log R^{1 / 2}\|f\|_{2}^{2} \\
R^{\gamma / 2} \log R^{1 / 2}\|f\|_{2}^{2}
\end{array} \lesssim\langle\mu\rangle_{\alpha}^{\frac{2}{q}} \times \begin{cases}R^{2 \beta(\alpha, q)+\varepsilon}\|f\|_{2}^{2}, & \text { if } \gamma \leq 0 \\
R^{2 \beta_{\circ}(\alpha, q)+\varepsilon}\|f\|_{2}^{2}, & \text { if } \gamma>0\end{cases}\right.
$$

From Lemma 3.3, we also see

$$
I I \lesssim\langle\mu\rangle_{\alpha}^{\frac{2}{q}} R^{2 \beta_{\circ}(\alpha, q)} \sum_{k}\left\|f_{k}^{j_{\circ}}\right\|_{2}^{2} \lesssim\langle\mu\rangle_{\alpha}^{\frac{2}{q}} R^{2 \beta_{\circ}(\alpha, q)}\|f\|_{2}^{2}
$$

Combining these estimates, we obtain that for $q \geq 2$, and for any $\epsilon>0$ and $R>1$,

$$
\|\widehat{f}\|_{L^{q}(d \mu)} \lesssim\langle\mu\rangle_{\alpha}^{\frac{1}{q}} R^{\widetilde{s}(\alpha, q, n)+\epsilon}\|f\|_{2}
$$

where $\widetilde{s}(\alpha, q, n)=\max \left\{\beta(\alpha, q), \beta_{\circ}(\alpha, q)\right\}$. Note that

$$
\widetilde{s}(\alpha, q, n)= \begin{cases}\max \left\{\frac{n}{2}-\frac{\alpha}{q}, \frac{n+1}{4}, \frac{3 n+1}{8}-\frac{\alpha}{4}\right\}, & \text { if } 0<\alpha \leq 1, \\ \max \left\{\frac{n}{2}-\frac{\alpha}{q}, \frac{n+1}{4}+\frac{1-\alpha}{2 q}, \frac{3 n+1}{8}-\frac{\alpha}{4}\right\}, & \text { if } 1<\alpha \leq n, \\ \max \left\{\frac{n}{2}-\frac{\alpha}{q}, \frac{n+1}{4}+\frac{n+1-2 \alpha}{2 q}, \frac{3 n+1}{8}-\frac{\alpha}{4}\right\}, & \text { if } n<\alpha \leq n+1 .\end{cases}
$$

If $3 \leq n<\alpha$, we can improve $\widetilde{s}(\alpha, q, n)$ slightly. In fact, we use Plancherel theorem and Lemma 2.5 so that, for $q \leq 2$, we have

$$
\|\widehat{f}\|_{L^{q}(d \mu)} \leq \mu\left(\mathbb{R}^{n+1}\right)^{\frac{1}{q}-\frac{1}{2}}\|\widehat{f}\|_{L^{2}(d \mu)} \lesssim\|\widehat{f}\|_{L^{2}}\left\|\left|\phi_{R}\right| * d \mu\right\|_{\infty}^{\frac{1}{2}} \leq R^{\frac{n+1-\alpha}{2}}\|f\|_{L^{2}}
$$

Since $\frac{3 n+1}{8}-\frac{\alpha}{4}>\frac{n+1-\alpha}{2}$ if $3 \leq n<\alpha \leq n+1$, it follows that, for $n<\alpha \leq n+1$

$$
\widetilde{s}(\alpha, q, n)=\max \left\{\frac{n}{2}-\frac{\alpha}{q}, \frac{n+1}{4}+\frac{n+1-2 \alpha}{2 q}, \frac{n+1-\alpha}{2}\right\} .
$$

This completes the proof. 
Proof of Theorem 1.2. We now show that if (7) holds for some $s=s_{0}$, then (3) holds for $s>s_{0}$. Since $\mu$ has compact support, by finite decomposition we may assume that $\mu$ is supported in $\overline{B(0,1)}$. Recall that the solution $u(x, t)$ is given by

$$
u(x, t)=\frac{1}{(2 \pi)^{n}} \int_{\mathbb{R}^{n}} e^{i x \cdot \xi} \cos (t|\xi|) \widehat{f}(\xi) d \xi+\frac{1}{(2 \pi)^{n}} \int_{\mathbb{R}^{n}} e^{i x \cdot \xi} \sin (t|\xi|) \frac{\widehat{g}(\xi)}{|\xi|} d \xi .
$$

Let $P_{j}$ be the projection operator $P_{j}$ for $j \geq 1$ given by $\widehat{P_{j} f}(\xi)=\beta\left(|\xi| / 2^{j}\right) \widehat{f}(\xi)$, where $\beta$ is a $C_{0}^{\infty}(\mathbb{R})$ function which is supported in $[1 / 2,2]$ and $\sum_{j \in \mathbb{Z}} \beta\left(|\xi| / 2^{j}\right)=1$ for $\xi \neq 0$. Also we define $P_{\leq 0} f$ such that $\widehat{P_{\leq 0} f}=\beta_{0} \widehat{f}$, where $\beta_{0}$ is a $C_{0}^{\infty}(\mathbb{R})$ function such that $\beta_{0}(|\xi|)=1-\sum_{j \geq 1} \beta\left(|\xi| / 2^{j}\right)$. We write

$$
u(x, t)=P_{\leq 0}(u(\cdot, t))(x)+\sum_{j \geq 1} P_{j}(u(\cdot, t))(x) .
$$

By Cauchy-Schwarz inequality and Plancherel theorem we have $\left|P_{\leq 0}(u(\cdot, t))(x)\right| \lesssim$ $\|f\|_{2}+\|g\|_{2}$. Since $\mu$ is supported in $\overline{B(0,1)}$, it follows that

$$
\left\|P_{\leq 0}(u(\cdot, t))\right\|_{L^{q}(d \mu)} \lesssim\|f\|_{2}+\|g\|_{2} .
$$

So, in order to show (3) for $s>s_{0}$ it is sufficient to show that (7) with $s=s_{0}$ implies

$$
\left\|P_{j}(u(\cdot, t))\right\|_{L^{q}(d \mu)} \lesssim 2^{s_{0} j}\|f\|_{2}+2^{\left(s_{0}-1\right) j}\|g\|_{2} .
$$

By (26) and time reversal symmetry this in turn follows from

$$
\left\|e^{i t \sqrt{-\Delta}} P_{j} h\right\|_{L^{q}(d \mu)} \leq 2^{s_{0} j}\|h\|_{2} .
$$

Since $\mu$ is supported in $\overline{B(0,1)}$, as before, using the smooth function $\eta$ satisfying $\eta \sim 1$ on $\overline{B(0,1)}$ and supp $\widehat{\eta} \subset B\left(0, \frac{1}{2}\right)$, we have

$$
\left\|e^{i t \sqrt{-\Delta}} P_{j} h\right\|_{L^{q}(d \mu)} \sim\left\|\eta e^{i t \sqrt{-\Delta}} P_{j} h\right\|_{L^{q}(d \mu)} .
$$

Note that the space time Fourier transform of $\eta e^{i t \sqrt{-\Delta}} P_{j} f(x)$ is supported in $\Gamma_{2^{j}}(1)$. Using (77) with $s=s_{0}$ and Plancherel theorem gives

$$
\left\|e^{i t \sqrt{-\Delta}} P_{j} h\right\|_{L^{q}(d \mu)} \lesssim 2^{s_{0} j}\left\|\eta e^{i t \sqrt{-\Delta}} P_{j} h\right\|_{2} \lesssim 2^{s_{0} j}\|h\|_{2} .
$$

Hence we get (21) and complete proof.

\section{Proof of Proposition 1.5}

Now, we obtain lower bounds on $s$ for which (7) may hold. This is done by constructing suitable functions and measures.

Firstly we show that if (7) holds, then

$$
s \geq \frac{n}{2}-\frac{\alpha}{q} .
$$


Let $\mu$ be the measure given by $d \mu(x)=\chi_{\overline{B(0,1)}}(x)|x|^{\alpha-n-1} d x$ and $f=\chi_{\Gamma_{R}(1)}$. Then, $\mu$ is obviously $\alpha$-dimensional and $|\widehat{f}(x)| \gtrsim R^{n}$ if $|x| \leq c R^{-1}$ with sufficiently small $c>0$. Hence (7) implies $R^{n} R^{-\alpha / q} \leq C R^{s} R^{\frac{n}{2}}$. So, letting $R \rightarrow \infty$ gives (28).

We now show the second condition:

$$
s \geq \begin{cases}\frac{n+1}{4}, & \text { if } 0<\alpha \leq 1 \\ \frac{n+1}{4}+\frac{1-\alpha}{2 q}, & \text { if } 1<\alpha \leq n \\ \frac{n+1}{4}+\frac{n+1-2 \alpha}{2 q}, & \text { if } n<\alpha \leq n+1 .\end{cases}
$$

Let us set

$$
P=\left\{\left(\xi_{1}, \xi^{\prime \prime}, \xi_{n+1}\right):\left|\xi_{1}-\xi_{n+1}\right| \leq \frac{1}{100},\left|\xi^{\prime \prime}\right| \leq \frac{\sqrt{R}}{100}, \frac{5}{4} R \leq\left|\xi_{1}+\xi_{n+1}\right| \leq \frac{3}{2} R\right\}
$$

Here $\xi^{\prime \prime} \in \mathbb{R}^{n-1}$. Then $P$ is contained in $\Gamma_{R}(1)$. Let $x=\left(x_{1}, x^{\prime \prime}, x_{n+1}\right)$ be the dual variables of $\left(\eta_{1}, \xi^{\prime \prime}, \eta_{n+1}\right)$, where $\eta_{1}=\left(\xi_{1}+\xi_{n+1}\right) / \sqrt{2}$ and $\eta_{n+1}=\left(\xi_{1}-\xi_{n+1}\right) / \sqrt{2}$.

For a given $\alpha$ let $\ell$ be a positive integer satisfying $\ell-1<\alpha \leq \ell$. We consider a measure $\mu$ defined by

$$
d \mu(x)=\prod_{i=1}^{n+1-\ell} d \delta\left(x_{i}\right)\left|x_{n-\ell+2}\right|^{\alpha-\ell} d x_{n-\ell+2} d x_{n-\ell+3} \cdots d x_{n+1},
$$

where $\delta$ is the 1 -dimensional delta measure and $x=\left(x_{1}, \ldots, x_{n+1}\right) \in \mathbb{R}^{n+1}$. If $n<$ $\alpha \leq n+1$, we set

$$
d \mu(x)=\left|x_{1}\right|^{\alpha-n-1} d x_{1} \cdots d x_{n+1} .
$$

Then it is easy to see that $\mu$ is $\alpha$-dimensional. In fact, considering the delta measure, $\mu\left(B\left(x_{0}, \rho\right)\right) \leq C \rho^{\alpha-\ell+1} \cdot \rho^{\ell-1}=C \rho^{\alpha}$ for any $\rho>0$ and $x_{0} \in \mathbb{R}^{n+1}$.

Let $f=\chi_{P}$. Then we have $\|f\|_{2} \lesssim R^{(n+1) / 4}$. We denote by $P^{*}$ the dual rectangle $(n-1)$ times

of $P$ of which dimensions are $C R^{-1} \times C R^{-1 / 2} \times \cdots \times C R^{-1 / 2} \times C$ for some constant $C$. It follows that $|\widehat{f}(x)| \gtrsim R^{1+(n-1) / 2}$ on a rectangle of which size is comparable to $P^{*}$. Hence, (17) gives

$$
R^{1+\frac{n-1}{2}} \mu\left(P^{*}\right)^{\frac{1}{q}} \lesssim R^{s+\frac{n+1}{4}} .
$$

Letting $R \rightarrow \infty$, we obtain (29) because

$$
\mu\left(P^{*}\right) \approx \begin{cases}1, & \text { if } 0<\alpha \leq 1 \\ R^{-\frac{1}{2}(\alpha-\ell+1)} \times R^{-\frac{1}{2}(\ell-2)}, & \text { if } 1<\alpha \leq n \\ R^{-(\alpha-n)} \times R^{-\frac{n-1}{2}}, & \text { if } n<\alpha \leq n+1 .\end{cases}
$$

Finally we show that (7) implies

$$
s \geq \begin{cases}\frac{n+2}{4}-\frac{\alpha}{4}, & \text { if } 1<\alpha \leq n, \\ \frac{n+1}{2}-\frac{\alpha}{2}, & \text { if } n<\alpha \leq n+1 .\end{cases}
$$


The condition (31) can be obtained by an adaptation of the example in [4] which was based on the one due to Wolff [26].

First we show (31) for $1<\alpha \leq n$. Let $\phi_{P}$ be a Schwartz function supported in $P$, where $P$ is given by (30). Let $N$ be an integer such that $R^{\frac{\alpha-1}{2}} \sim N$, and let $v_{1}, \ldots, v_{N}$ be the lattice points which are contained in $B_{n-1}(0,1)$ and separated by distance $\sim R^{-\frac{\alpha-1}{2(n-1)}}$. Now we consider a Schwartz function $F$ supported in $\Gamma_{R}(1)$, which is given by

$$
F\left(\eta_{1}, \xi^{\prime \prime}, \eta_{n+1}\right)=N^{-\frac{1}{2}} \sum_{k=1}^{N} \phi_{P}(\xi) e^{i v_{k} \cdot \xi^{\prime \prime}} .
$$

Here again, $\eta_{1}$ and $\eta_{n+1}$ are the coordinates defined as in the above. Note that $\widehat{F}$ is a sum of translations of $\widehat{\phi_{P}}$, i.e. $\widehat{F}=N^{-1 / 2} \sum_{k=1}^{N} \widehat{\phi_{P}}\left(x_{1}, x^{\prime \prime}-v_{k}, x_{n+1}\right)$. Since $1<\alpha \leq n$, we see $R^{-\frac{\alpha-1}{2(n-1)}} \geq R^{-\frac{1}{2}}$, which implies that $P^{*}+v_{k}$ 's are almost disjoint. By rapid decay of $\widehat{\phi_{P}}$ outside of $P^{*}$ we see that $|\widehat{F}| \gtrsim N^{-\frac{1}{2}} R^{\frac{n+1}{2}} \sim R^{\frac{2 n+3-\alpha}{4}}$ on $S:=\bigcup_{k=1}^{N}\left(P^{*}+v_{k}\right)$. Consider the measure $d \mu=R^{\frac{n+2-\alpha}{2}} \chi_{S} d x$, which is an $\alpha$ dimensional measure with $\langle\mu\rangle_{\alpha} \lesssim 1$ when $1<\alpha \leq n$. In fact, for $R^{-1} \leq \rho<R^{-1 / 2}$ and $x_{\circ} \in \mathbb{R}^{n+1}$ it is easy to see

$$
\mu\left(B\left(x_{\circ}, \rho\right)\right) \leq R^{\frac{n+2-\alpha}{2}}\left|S \cap B\left(x_{\circ}, \rho\right)\right| \leq R^{\frac{n+2-\alpha}{2}} R^{-1} \rho^{n} \leq \rho^{-(n-\alpha)} \rho^{n}=\rho^{\alpha} .
$$

The other cases $\rho<R^{-1}, R^{-1 / 2}<\rho \leq 1$, and $\rho>1$ can be treated similarly. Note that $\int_{S} d \mu \sim 1$ and $\|F\|_{2}^{2} \leq N^{-1} \sum_{k=1}^{N}\left\|\phi_{P}\right\|_{2}^{2} \leq C R^{\frac{n+1}{4}}$. Hence (7) implies $R^{\frac{n+2-\alpha}{2}} \lesssim R^{s}$, which gives (31).

Now we proceed to show (31) for $\alpha>n$. Similarly as before, let $u_{1}, \ldots, u_{M}$ be the lattice points which are contained in $B_{n-1}(0,1)$ and separated by about $R^{-\frac{2 \alpha-n-1}{2(n+1)}} \gg$ $R^{-\frac{1}{2}}$ so that $M \sim R^{\frac{(n-1)(2 \alpha-n-1)}{2(n+1)}}$. Under the same assumption, let $w_{1}, \ldots, w_{L}$ be the lattice points which are contained in $(-1 / 100,1 / 100)$ and separated by $R^{-\frac{2 \alpha-n-1}{n+1}}$ such that $L \sim R^{\frac{2 \alpha-n-1}{n+1}}$. We set

$$
G\left(\eta_{1}, \xi^{\prime \prime}, \eta_{n+1}\right)=(M L)^{-\frac{1}{2}} \sum_{k=1}^{M} \sum_{j=1}^{L} \phi_{P}(\xi) e^{i\left(u_{k} \cdot \xi^{\prime \prime}+w_{j} \eta_{n+1}\right)}
$$

Hence it follows that $|\widehat{G}| \gtrsim(M L)^{-\frac{1}{2}} R^{\frac{n+1}{2}}=R^{\frac{3 n+3-2 \alpha}{4}}$ on $T=\bigcup_{k=1}^{M} \bigcup_{j=1}^{L}\left(P^{*}+u_{k}+w_{j}\right)$. We now consider an $\alpha$-dimensional measure $d \mu=R^{n+1-\alpha} \chi_{T} d x$. Noting $\int_{T} d \mu \sim 1$ and $\|G\|_{2} \sim R^{\frac{n+1}{4}}$, we get the second condition in (31) by letting $R \rightarrow \infty$.

\section{REFERENCES}

[1] J. Bennett, A. Carbery, and T. Tao. On the multilinear restriction and kakeya conjectures. Acta Math., 196(2):261-302, 2006.

[2] J. Bourgain. Hausdorff dimension and distance sets. Israel J. Math., 87(1-3):193-201, 1994.

[3] J. Bourgain and L. Guth. Bounds on oscillatory integral operators based on multilinear estimates. Geom. Funct. Anal., 21(6):1239-1295, 2011. 
[4] M. B. Erdoğan. A note on the fourier transform of fractal measures. Math. Res. Lett., 11(23):299-314, 2004.

[5] M. B. Erdoğan. A bilinear fourier extension theorem and applications to the distance set problem. Int. Math. Res. Not., 2005(23):1411-1425, 2005.

[6] M. B. Erdoğan. On falconer's distance set conjecture. Rev. Mat. Iberoam., 22(2):649-662, 2006.

[7] D. Fang and C. Wang. Some remarks on strichartz estimates for homogeneous wave equation. Nonlinear Anal., 65(3):697-706, 2006.

[8] J. Ginibre and G. Velo. The global cauchy problem for the non linear klein-gordon equation. Math. Z., 189(4):487-505, 1985.

[9] L. Guth. A restriction estimate using polynomial partitioning. J. Amer. Math. Soc., 29(2):371$413,2016$.

[10] S. Ham and S. Lee. Restriction estimates for space curves with respect to general measures. Adv. Math., 254:251-279, 2014.

[11] M. Keel and T. Tao. Endpoint strichartz estimates. Amer J. Math., 120(5):955-980, 1998.

[12] S. Klainerman and M. Machedon. Space-time estimates for null forms and the local existence theorem. Comm. Pure Appl. Math., 46(9):1221-1268, 1993.

[13] S. Lee. Bilinear restriction estimates for surfaces with curvatures of different signs. Trans. Amer. Math. Soc., 358(8):3511-3533, 2006.

[14] H. Lindblad and C. D. Sogge. On existence and scattering with minimal regularity for semilinear wave equations. J. Funct. Anal., 130(2):357-426, 1995.

[15] R. Lucà and K. Rogers. Average decay of the fourier transform of measures with applications. to appear in J. Eur. Math. Soc., 2015.

[16] P. Mattila. Spherical averages of fourier transforms of measures with finite energy; dimensions of intersections and distance sets. Mathematika, 34(02):207-228, 1987.

[17] T. Mitsis. On a problem related to sphere and circle packing. J. London Math. Soc., 60(2):501$516,1999$.

[18] S. Montgomery-Smith. Time decay for the bounded mean oscillation of solutions of the schrödinger and wave equations. Duke Math. J., 91(2):393-408, 1998.

[19] D. Oberlin. Packing spheres and fractal strichartz estimates in $\backslash^{d}$ for $d \geq 3$. Proc. Amer. Math. Soc., 134(11):3201-3209, 2006.

[20] D. Oberlin. Unions of hyperplanes, unions of spheres, and some related estimates. Illinois J. Math., 51(4):1265-1274, 2007.

[21] H. Pecher. Nonlinear small data scattering for the wave and klein-gordon equation. Math. Z., 185(2):261-270, 1984.

[22] R. Strichartz. Restrictions of fourier transforms to quadratic surfaces and decay of solutions of wave equations. Duke Math. J., 44(3):705-714, 1977.

[23] T. Tao. A sharp bilinear restriction estimate for paraboloids. Geom. Funct. Anal., 13(6):1359$1384,2003$.

[24] T. Tao and A. Vargas. A bilinear approach to cone multipliers i, ii. Geom. Funct. Anal., $10(1): 185-258,2000$.

[25] T. Tao, A. Vargas, and L. Vega. A bilinear approach to the restriction and kakeya conjectures. J. Amer. Math. Soc., 11(4):967-1000, 1998.

[26] T. Wolff. Decay of circular means of fourier transforms of measures. Int. Math. Res. Not., 1999(10):547-567, 1999.

[27] T. Wolff. Local smoothing type estimates on $l^{p}$ for large p. Geom. Funct. Anal., 10(5):1237$1288,2000$.

[28] T. Wolff. A sharp bilinear cone restriction estimate. Ann. Math., 153(3):661-698, 2001. 
School of Mathematical Sciences, Seoul National University, Seoul 08826, RePUBLIC OF KOREA

E-mail address: akilus@snu.ac.kr

School of Mathematics, Korea Institute for Advanced Study, Seoul 02455, RePUBLIC OF KOREA

E-mail address: hamsh@kias.re.kr

School of Mathematical Sciences, Seoul National University, Seoul 08826, RePUBLIC OF KOREA

E-mail address: shklee@snu.ac.kr 